\title{
Effect of Storage Periods on Seed Quality Parameters of Buckwheat (Fagopyrum esculenum Moench) Genotypes
}

\author{
Ajay Kumar Pandey ${ }^{1 *}$, A. K. Chaurasia ${ }^{1}$ and Dhananjay Tiwari ${ }^{2}$ \\ ${ }^{1}$ Department of G.P.B., ${ }^{2}$ Department of Agronomy, SHUATS, Prayagraj (U.P.), India \\ *Corresponding author
}

\section{A B S T R A C T}

\begin{tabular}{|l|}
\hline Key w o r d s \\
Quality parameter, \\
$\begin{array}{l}\text { Decline, Storage } \\
\text { period and Vigour } \\
\text { index }\end{array}$ \\
\hline Article Info \\
\hline $\begin{array}{l}\text { Accepted: } \\
\text { 24 July 2020 } \\
\text { Available Online: } \\
\text { 10 August } 2020\end{array}$ \\
\hline
\end{tabular}

\section{Introduction}

Common buckwheat (Fagopyrum esculentum Moench) has been a crop of secondary importance in many countries and presently cultivated in every cereals growing country in the world. Buckwheat is one of the traditional crops cultivated in Asia, Central and Eastern Europe (Wijngaard and Arendt, 2006).About $90 \%$ of cultivated lands in the Himalayas are occupied by buckwheat and it is the only crop grown at the height of $4500 \mathrm{~m}$ and is a staple crop for people of those areas. Buckwheat, having its name mostly similar to that of common bread wheat (Triticum aestivum), is completely different from the bread wheat
(Rana et al., 2014).About $90 \%$ of cultivated lands in the Himalayas are occupied by buckwheat (Iqbal et al., 2003). AICRP on under exploited and unexploited crops research programme considered the importance of buckwheat and recognized it as a miracle-crop in 1982 (Horie et al., 2012).

Seed deterioration during storage is a gradual and inevitable process causing considerable losses. Seed tends to lose viability and vigour during storage and information on storability of seed lots from harvest until the next planting season and also for carry over purposes is of immense importance in any seed production programme (Gupta and 
Aneja, 2001). During storage, number of biotic and a biotic factors influence the storage potential of seeds and results in gradual seed deterioration and ultimately death of the seeds (Kumar et al., 2014). Seed mycoflora has been recognized as an important factor responsible for deterioration in quality of seeds during storage.

The seed potentiality is mainly achieved by treating the seeds with various chemicals and botanicals that can reduce the infestation and maintain the quality of the seed in terms of viability and vigour for longer periods in storage (Wani et al., 2014).To maintain the quality of seeds during storage the standardization of suitable seed treatments and packaging material is most important because seed treatment is the basic measure to assure adequately healthy crops at emergence and during further growth of plants (Suzuki et al., 2012).

\section{Materials and Methods}

A lab experiment was carried out in Seed Testing Laboratory of Department of Genetics and Plant Breeding, Sam Higginbotton University of Agriculture, Technology and Sciences (SHUATS), Naini, Prayagraj.

Twenty genotypes of buckwheat were used in the present investigation. Among them one genotype was obtained from ICARVivekananda Parvatiya Krishi Anusandhan Sansthan, Almora (Uttarakhand), four from Mountain Agriculture Research and Extension Centre Sangla, (Kinnaur) (Palampur, Himachal Pradesh), five from ICAR-National Organic Farming Research Institute, Gangtok (Sikkim) and the remaining 10 from ICAR-National Bureau of Plant Genetics Resources, New Delhi.

During February - March 2019, freshly ripened seeds of buckwheat were collected from 20 genotypes that were grown in Seed Research Farm at SHUATS. Seeds from all genotypes were stored at normal temperature and tested for every three months interval for germination $(\%)$, total seedling length $(\mathrm{cm})$, seedling dry weight (g) and seed vigour Indices (I and II).Seed vigour Indices I and II are obtained by multiplying Germination (\%) and Seedling length $(\mathrm{cm})$ and Germination (\%) and Seedling dry weight (g) respectively (ISTA, 2010).

\section{Results and Discussion}

All the genotypes investigated for quality parameters displayed considerable amount of differences in their mean performances with respect to all studied characters.

The Table 1 indicates that the genotypes $G_{7}$ and $\mathrm{G}_{17}$ recorded highest germination $(\%)$ of 79.25 after 12 months storage period. The lowest germination $(66.00 \%)$ after 12 months storage period was recorded by $\mathrm{G}_{12}$. The Table 1 indicates that the maximum seedling length $(18.40 \mathrm{~cm})$ was recorded by the genotype $\mathrm{G}_{2}$ and the minimum $(13.41 \mathrm{~cm})$ was recorded by $\mathrm{G}_{20}$ after 12 months storage period.

The Table 2 indicates that the maximum seedling dry weight was recorded by $G_{18}$ genotype $(0.07 \mathrm{~g})$. The minimum seedling dry weight $(0.04 \mathrm{~g})$ was recorded by the genotypes $\mathrm{G}_{1}, \mathrm{G}_{2}, \mathrm{G}_{3}, \mathrm{G}_{4}, \mathrm{G}_{7}, \mathrm{G}_{11}, \mathrm{G}_{12}$ and $\mathrm{G}_{13}$. The Table 2 also indicates that after 12 months storage period, the genotype $G_{13}$ recorded the highest seed vigour index-I (1419.33) and the lowest (1092.90) was recorded by genotype $\mathrm{G}_{20}$.

The Table 3 indicates that the highest vigour Index-II after 12 months storage period was recorded by genotype $\mathrm{G}_{18}$ (5.32) and lowest (2.47) by the genotype $\mathrm{G}_{12}$. 
Table.1 Mean performance of germination (\%) and seedling length $(\mathrm{cm})$

\begin{tabular}{|c|c|c|c|c|c|c|c|c|c|}
\hline \multirow[t]{2}{*}{ S. No. } & \multirow[t]{2}{*}{ Genotypes } & \multicolumn{4}{|c|}{ Germination (\%) } & \multicolumn{4}{|c|}{ Seedling length $(\mathrm{cm})$} \\
\hline & & $\begin{array}{c}3 \\
\text { MAS }^{*}\end{array}$ & $\begin{array}{c}6 \\
\text { MAS }\end{array}$ & $\begin{array}{c}9 \\
\text { MAS }\end{array}$ & $\begin{array}{c}12 \\
\text { MAS }\end{array}$ & $\begin{array}{c}3 \\
\text { MAS }\end{array}$ & $\begin{array}{c}6 \\
\text { MAS }\end{array}$ & $\begin{array}{c}9 \\
\text { MAS }\end{array}$ & $\begin{array}{c}12 \\
\text { MAS }\end{array}$ \\
\hline 1 & $\mathrm{G}_{1}$ & 97.50 & 88.00 & 97.50 & 88.00 & 97.50 & 88.00 & 97.50 & 88.00 \\
\hline 2 & $\mathrm{G}_{2}$ & 95.25 & 80.75 & 95.25 & 80.75 & 95.25 & 80.75 & 95.25 & 80.75 \\
\hline 3 & $\mathrm{G}_{3}$ & 93.25 & 91.50 & 93.25 & 91.50 & 93.25 & 91.50 & 93.25 & 91.50 \\
\hline 4 & $\mathrm{G}_{4}$ & 95.25 & 82.00 & 95.25 & 82.00 & 95.25 & 82.00 & 95.25 & 82.00 \\
\hline 5 & $\mathrm{G}_{5}$ & 97.50 & 87.50 & 97.50 & 87.50 & 97.50 & 87.50 & 97.50 & 87.50 \\
\hline 6 & $\mathrm{G}_{6}$ & 97.75 & 87.75 & 97.75 & 87.75 & 97.75 & 87.75 & 97.75 & 87.75 \\
\hline 7 & $\mathrm{G}_{7}$ & 95.00 & 90.00 & 95.00 & 90.00 & 95.00 & 90.00 & 95.00 & 90.00 \\
\hline 8 & $\mathrm{G}_{8}$ & 96.75 & 90.75 & 96.75 & 90.75 & 96.75 & 90.75 & 96.75 & 90.75 \\
\hline 9 & $\mathrm{G}_{9}$ & 97.50 & 89.50 & 97.50 & 89.50 & 97.50 & 89.50 & 97.50 & 89.50 \\
\hline 10 & $\mathrm{G}_{10}$ & 97.00 & 89.50 & 97.00 & 89.50 & 97.00 & 89.50 & 97.00 & 89.50 \\
\hline 11 & $\mathrm{G}_{11}$ & 92.00 & 89.50 & 92.00 & 89.50 & 92.00 & 89.50 & 92.00 & 89.50 \\
\hline 12 & $\mathrm{G}_{12}$ & 95.75 & 83.00 & 95.75 & 83.00 & 95.75 & 83.00 & 95.75 & 83.00 \\
\hline 13 & $\mathrm{G}_{13}$ & 90.50 & 89.75 & 90.50 & 89.75 & 90.50 & 89.75 & 90.50 & 89.75 \\
\hline 14 & $\mathrm{G}_{14}$ & 92.50 & 86.25 & 92.50 & 86.25 & 92.50 & 86.25 & 92.50 & 86.25 \\
\hline 15 & $\mathrm{G}_{15}$ & 91.75 & 86.00 & 91.75 & 86.00 & 91.75 & 86.00 & 91.75 & 86.00 \\
\hline 16 & $\mathrm{G}_{16}$ & 92.25 & 86.25 & 92.25 & 86.25 & 92.25 & 86.25 & 92.25 & 86.25 \\
\hline 17 & $\mathrm{G}_{17}$ & 97.50 & 92.25 & 97.50 & 92.25 & 97.50 & 92.25 & 97.50 & 92.25 \\
\hline 18 & $\mathrm{G}_{18}$ & 94.00 & 92.50 & 94.00 & 92.50 & 94.00 & 92.50 & 94.00 & 92.50 \\
\hline 19 & $\mathrm{G}_{19}$ & 97.00 & 93.50 & 97.00 & 93.50 & 97.00 & 93.50 & 97.00 & 93.50 \\
\hline \multirow[t]{5}{*}{20} & $\mathrm{G}_{20}$ & 97.00 & 91.75 & 97.00 & 91.75 & 97.00 & 91.75 & 97.00 & 91.75 \\
\hline & C.D. & 2.537 & 5.889 & 2.537 & 5.889 & 2.537 & 5.889 & 2.537 & 5.889 \\
\hline & $\mathrm{SE}(\mathrm{m})$ & 0.894 & 2.077 & 0.894 & 2.077 & 0.894 & 2.077 & 0.894 & 2.077 \\
\hline & SE(d) & 1.265 & 2.937 & 1.265 & 2.937 & 1.265 & 2.937 & 1.265 & 2.937 \\
\hline & C.V. & 1.88 & 4.698 & 1.88 & 4.698 & 1.88 & 4.698 & 1.88 & 4.698 \\
\hline
\end{tabular}

*MAS=Month after storage

Table.2 Mean performance table for seedling dry weight $(\mathrm{g})$ and seed vigour Index-I

\begin{tabular}{|l|l|l|l|l|l|l|l|l|l|}
\hline S. No. & Genotypes & \multicolumn{4}{|c|}{ Seedling dry weight (g) } & \multicolumn{4}{c|}{ Seed Vigour Index -I } \\
\hline & & 3 & 6 MAS & 9 MAS & 12 & 3 MAS & 6 MAS & 9 MAS & 12 \\
& & MAS & & & MAS & & & & MAS \\
\hline $\mathbf{1}$ & $\mathrm{G}_{1}$ & 0.06 & 0.06 & 0.06 & 0.06 & 0.06 & 0.06 & 0.06 & 0.06 \\
\hline $\mathbf{2}$ & $\mathrm{G}_{2}$ & 0.07 & 0.06 & 0.07 & 0.06 & 0.07 & 0.06 & 0.07 & 0.06 \\
\hline $\mathbf{3}$ & $\mathrm{G}_{3}$ & 0.08 & 0.06 & 0.08 & 0.06 & 0.08 & 0.06 & 0.08 & 0.06 \\
\hline $\mathbf{4}$ & $\mathrm{G}_{4}$ & 0.09 & 0.07 & 0.09 & 0.07 & 0.09 & 0.07 & 0.09 & 0.07 \\
\hline $\mathbf{5}$ & $\mathrm{G}_{5}$ & 0.08 & 0.07 & 0.08 & 0.07 & 0.08 & 0.07 & 0.08 & 0.07 \\
\hline $\mathbf{6}$ & $\mathrm{G}_{6}$ & 0.09 & 0.07 & 0.09 & 0.07 & 0.09 & 0.07 & 0.09 & 0.07 \\
\hline $\mathbf{7}$ & $\mathrm{G}_{7}$ & 0.07 & 0.06 & 0.07 & 0.06 & 0.07 & 0.06 & 0.07 & 0.06 \\
\hline $\mathbf{8}$ & $\mathrm{G}_{8}$ & 0.09 & 0.07 & 0.09 & 0.07 & 0.09 & 0.07 & 0.09 & 0.07 \\
\hline $\mathbf{9}$ & $\mathrm{G}_{9}$ & 0.09 & 0.07 & 0.09 & 0.07 & 0.09 & 0.07 & 0.09 & 0.07 \\
\hline
\end{tabular}




\begin{tabular}{|l|l|l|l|l|l|l|l|l|l|}
\hline $\mathbf{1 0}$ & $\mathrm{G}_{10}$ & 0.09 & 0.08 & 0.09 & 0.08 & 0.09 & 0.08 & 0.09 & 0.08 \\
\hline $\mathbf{1 1}$ & $\mathrm{G}_{11}$ & 0.06 & 0.05 & 0.06 & 0.05 & 0.06 & 0.05 & 0.06 & 0.05 \\
\hline $\mathbf{1 2}$ & $\mathrm{G}_{12}$ & 0.06 & 0.06 & 0.06 & 0.06 & 0.06 & 0.06 & 0.06 & 0.06 \\
\hline $\mathbf{1 3}$ & $\mathrm{G}_{13}$ & 0.07 & 0.06 & 0.07 & 0.06 & 0.07 & 0.06 & 0.07 & 0.06 \\
\hline $\mathbf{1 4}$ & $\mathrm{G}_{14}$ & 0.09 & 0.07 & 0.09 & 0.07 & 0.09 & 0.07 & 0.09 & 0.07 \\
\hline $\mathbf{1 5}$ & $\mathrm{G}_{15}$ & 0.09 & 0.08 & 0.09 & 0.08 & 0.09 & 0.08 & 0.09 & 0.08 \\
\hline $\mathbf{1 6}$ & $\mathrm{G}_{16}$ & 0.10 & 0.08 & 0.10 & 0.08 & 0.10 & 0.08 & 0.10 & 0.08 \\
\hline $\mathbf{1 7}$ & $\mathrm{G}_{17}$ & 0.06 & 0.06 & 0.06 & 0.06 & 0.06 & 0.06 & 0.06 & 0.06 \\
\hline $\mathbf{1 8}$ & $\mathrm{G}_{18}$ & 0.10 & 0.09 & 0.10 & 0.09 & 0.10 & 0.09 & 0.10 & 0.09 \\
\hline $\mathbf{1 9}$ & $\mathrm{G}_{19}$ & 0.10 & 0.08 & 0.10 & 0.08 & 0.10 & 0.08 & 0.10 & 0.08 \\
\hline $\mathbf{2 0}$ & $\mathrm{G}_{20}$ & 0.09 & 0.08 & 0.09 & 0.08 & 0.09 & 0.08 & 0.09 & 0.08 \\
\hline & C.D. & N/A & N/A & N/A & N/A & N/A & N/A & N/A & N/A \\
\hline & SE(m) & 0.012 & 0.01 & 0.012 & 0.01 & 0.012 & 0.01 & 0.012 & 0.01 \\
\hline & SE(d) & 0.017 & 0.015 & 0.017 & 0.015 & 0.017 & 0.015 & 0.017 & 0.015 \\
\hline & C.V. & 30.936 & 30.36 & 30.936 & 30.36 & 30.936 & 30.36 & 30.936 & 30.36 \\
\hline
\end{tabular}

*MAS=Month after storage

Table.3 Mean performance table for seed vigour Index-II

\begin{tabular}{|c|c|c|c|c|c|}
\hline \multirow[t]{2}{*}{ S. No } & \multirow[t]{2}{*}{ Genotypes } & \multicolumn{4}{|c|}{ Seed Vigour Index -II } \\
\hline & & $3 \mathrm{MAS}^{*}$ & $6 \mathrm{MAS}$ & 9 MAS & $12 \mathrm{MAS}$ \\
\hline 1 & $\mathrm{G}_{1}$ & 5.85 & 5.85 & 5.85 & 5.85 \\
\hline 2 & $\mathrm{G}_{2}$ & 6.43 & 6.43 & 6.43 & 6.43 \\
\hline 3 & $\mathrm{G}_{3}$ & 7.44 & 7.44 & 7.44 & 7.44 \\
\hline 4 & $\mathrm{G}_{4}$ & 8.10 & 8.10 & 8.10 & 8.10 \\
\hline 5 & $\mathrm{G}_{5}$ & 7.81 & 7.81 & 7.81 & 7.81 \\
\hline 6 & $\mathrm{G}_{6}$ & 8.79 & 8.79 & 8.79 & 8.79 \\
\hline 7 & $\mathrm{G}_{7}$ & 6.17 & 6.17 & 6.17 & 6.17 \\
\hline 8 & $\mathrm{G}_{8}$ & 8.70 & 8.70 & 8.70 & 8.70 \\
\hline 9 & $\mathrm{G}_{9}$ & 8.77 & 8.77 & 8.77 & 8.77 \\
\hline 10 & $\mathrm{G}_{10}$ & 8.74 & 8.74 & 8.74 & 8.74 \\
\hline 11 & $\mathrm{G}_{11}$ & 5.53 & 5.53 & 5.53 & 5.53 \\
\hline 12 & $\mathrm{G}_{12}$ & 5.76 & 5.76 & 5.76 & 5.76 \\
\hline 13 & $\mathrm{G}_{13}$ & 6.33 & 6.33 & 6.33 & 6.33 \\
\hline 14 & $\mathrm{G}_{14}$ & 8.08 & 8.08 & 8.08 & 8.08 \\
\hline 15 & $\mathrm{G}_{15}$ & 7.80 & 7.80 & 7.80 & 7.80 \\
\hline 16 & $\mathrm{G}_{16}$ & 8.96 & 8.96 & 8.96 & 8.96 \\
\hline 17 & $\mathrm{G}_{17}$ & 5.85 & 5.85 & 5.85 & 5.85 \\
\hline 18 & $\mathrm{G}_{18}$ & 9.12 & 9.12 & 9.12 & 9.12 \\
\hline 19 & $\mathrm{G}_{19}$ & 9.48 & 9.48 & 9.48 & 9.48 \\
\hline \multirow[t]{5}{*}{20} & $\mathrm{G}_{20}$ & 8.49 & 8.49 & 8.49 & 8.49 \\
\hline & C.D. & N/A & N/A & N/A & N/A \\
\hline & $\mathrm{SE}(\mathrm{m})$ & 1.179 & 1.179 & 1.179 & 1.179 \\
\hline & $\mathrm{SE}(\mathrm{d})$ & 1.668 & 1.668 & 1.668 & 1.668 \\
\hline & C.V. & 31.001 & 31.001 & 31.001 & 31.001 \\
\hline
\end{tabular}

*MAS=Month after storage 
In conclusion the Proper storage of seed is an important sequel after harvesting. The successful cultivation of any crop is largely dependent on viability of the seed during storage and the ability to germinate.

The overall performance of genotypes under study judged on the basis of results obtained indicated that there was a decline in seed quality parameters with increased duration of storage. Many genotypes displayed a huge difference among the studied parameters as the storage time progressed. The interaction of moisture, temperature, initial seed quality and even specific genotype resulted for variability in studied parameters.

\section{References}

Gupta, A. and Aneja, K.R. (2001).Mycofloral Spectrum during Storage and its Effect on Seed Viability of Soybean [Glycine $\max ($ L.) Merrill] Seeds Under Ambient Conditions. Proceedings of National Academy of Sciences, India 71 B III \&IV: 245-253.

Horie, T., Karahara, I. and Katsuhara, M. (2012). Salinity Tolerance mechanisms in Glycophytes: An Overview with the central focus on Rice Plants. Rice (NY) 5:11 doi: 10.1186/1939-8433-5-11.

Iqbal, Z., Hiradate, S., Noda, A., Isojima, S. and Fuji, Y. (2003). Allelopathic Activity of Buckwheat: Isolation and Characterization of Phenolics. Weed Science, 657-662.

Kumar, T.P., Asha, A.M., Maruthi, J.B., Vishwanath, K. (2014). Influence of Seed Treatment Chemicals and Containers on Seed Quality of Marigold During Storage. The Bioscan, 9:937942.

Rana, S., Sood, P. and Rana, M.C. (2004). Integrated Weed Management in Buckwheat. Fagopyrum. 21:108-113.

Suzuki, T., Mukasa, Y., Morishita, T., Takigawa, S. and Noda, T. (2012). Traits of Shattering Resistant Buckwheat "W/SK86GF". Breeding Science, 62:360-364.

Wani, A.A., Joshi, J., Titov, A., Tomar, D.S. (2014). Effect of Seed Treatments and Packing Materials on Seed Quality Parameters of Maize (Zea mays L.) During Storage. India Journal of Applied Research. 4(4):102-108.

Wijngaard, H.H. and Arendt, E.K. (2006). Buckwheat. Cereal Chemistry, 83(4):391-401.

International Seed Testing Association (2010). International Rules for Seed Testing. ISTA, Bassersdorf, Switzerland.

\section{How to cite this article:}

Ajay Kumar Pandey, A. K. Chaurasia and Dhananjay Tiwari. 2020. Effect of Storage Periods on Seed Quality Parameters of Buckwheat (Fagopyrum esculenum Moench) Genotypes. Int.J.Curr.Microbiol.App.Sci. 9(08): 3098-3102. doi: https://doi.org/10.20546/ijcmas.2020.908.351 